\title{
New concepts of fractional quantum calculus and applications to impulsive fractional $q$-difference equations
}

\author{
Jessada Tariboon ${ }^{1 *}$, Sotiris K Ntouyas ${ }^{2}$ and Praveen Agarwal ${ }^{3}$
}

*Correspondence:

jessadat@kmutnb.ac.th

${ }^{1}$ Nonlinear Dynamic Analysis

Research Center, Department of

Mathematics, Faculty of Applied

Science, King Mongkut's University

of Technology North Bangkok,

Bangkok, 10800, Thailand

Full list of author information is

available at the end of the article

\begin{abstract}
In this paper we define new concepts of fractional quantum calculus by defining a new $q$-shifting operator. After giving the basic properties we define the $q$-derivative and $q$-integral. New definitions of Riemann-Liouville fractional $q$-integral and $q$-difference on an interval $[a, b]$ are given and their basic properties are discussed. As applications of the new concepts, we prove existence and uniqueness results for first and second order initial value problems for impulsive fractional $q$-difference equations.
\end{abstract}

MSC: $26 \mathrm{A33} ; 39 \mathrm{~A} 13 ; 34 \mathrm{~A} 37$

Keywords: quantum calculus; impulsive fractional q-difference equations; existence; uniqueness

\section{Introduction}

The quantum calculus is known as the calculus without limits. It substitutes the classical derivative by a difference operator, which allows one to deal with sets of nondifferentiable functions. Quantum difference operators have an interest role due to their applications in several mathematical areas such as orthogonal polynomials, basic hypergeometric functions, combinatorics, the calculus of variations, mechanics and the theory of relativity. The book by Kac and Cheung [1] covers many of the fundamental aspects of the quantum calculus.

In recent years, the topic of $q$-calculus has attracted the attention of several researchers and a variety of new results can be found in the papers [2-20] and the references cited therein.

In [21] the notions of $q_{k}$-derivative and $q_{k}$-integral of a function $f: J_{k}:=\left[t_{k}, t_{k+1}\right] \rightarrow \mathbb{R}$, have been introduced and their basic properties was proved. As applications existence and uniqueness results for initial value problems for first and second order impulsive $q_{k}$ difference equations are proved. $q$-calculus analogs of some classical integral inequalities, such as the Hölder, Hermite-Hadamard, Trapezoid, Ostrowski, Cauchy-BunyakovskySchwarz, Grüss, and Grüss-Čebyšev ones are proved in [22].

In this paper we define new concepts of fractional quantum calculus by defining a new $q$-shifting operator ${ }_{a} \Phi_{q}(m)=q m+(1-q) a$. After giving the basic properties we define the $q$-derivative and $q$-integral. New definitions of Riemann-Liouville fractional $q$-integral 
and $q$-difference on an interval $[a, b]$ are given and their basic properties are discussed. As applications of the new concepts, we prove existence and uniqueness results for first and second order initial value problems for impulsive fractional $q$-difference equations.

In applications, as in constructing a $q$-Taylor formula or solving $q$-differential equations and inequalities of fractional order, it is interesting to allow nonzero lower limits of $q$ integration. In $[23,24]$, the authors studied fractional $q$-integrals and $q$-derivatives which are based on Jackson sense on interval $[a, b]$. However, if the upper and lower limits of $q$-integration are $b$ and $a=b q^{n}$ for some $n \in \mathbb{N}$, respectively, then the infinite sum of the definition of $q$-integration is reduced to a finite sum, which is the restriction of definition of $q$-integral. From this point, our results generalized the classical definitions of fractional $q$-integrals and $q$-derivatives by shifting the point of the origin from zero to be a constant $a \in \mathbb{R}$ and applying the results to establish the impulsive fractional quantum difference equations.

\section{Preliminaries}

To make this paper self-contained, below we recall some known facts on fractional $q$-calculus. The presentation here can be found in, for example, [6,7].

For $q \in(0,1)$, define

$$
[m]_{q}=\frac{1-q^{m}}{1-q}, \quad m \in \mathbb{R}
$$

The $q$-analog of the power function $(n-m)^{k}$ with $k \in \mathbb{N}_{0}:=\{0,1,2, \ldots\}$ is

$$
(n-m)^{(0)}=1, \quad(n-m)^{(k)}=\prod_{i=0}^{k-1}\left(n-m q^{i}\right), \quad k \in \mathbb{N}, n, m \in \mathbb{R} .
$$

More generally, if $\gamma \in \mathbb{R}$, then

$$
(n-m)^{(\gamma)}=\prod_{i=0}^{\infty} \frac{n-m q^{i}}{n-m q^{\gamma+i}}, \quad n \neq 0 .
$$

Note if $m=0$, then $n^{(\gamma)}=n^{\gamma}$. We also use the natation $0^{(\gamma)}=0$ for $\gamma>0$. The $q$-gamma function is defined by

$$
\Gamma_{q}(t)=\frac{(1-q)^{(t-1)}}{(1-q)^{t-1}}, \quad t \in \mathbb{R} \backslash\{0,-1,-2, \ldots\} .
$$

Obviously, $\Gamma_{q}(t+1)=[t]_{q} \Gamma_{q}(t)$.

The $q$-derivative of a function $h$ is defined by

$$
\left(D_{q} h\right)(t)=\frac{h(t)-h(q t)}{(1-q) t} \quad \text { for } t \neq 0 \quad \text { and } \quad\left(D_{q} h\right)(0)=\lim _{t \rightarrow 0}\left(D_{q} h\right)(t)
$$

and the $q$-derivatives of higher order are given by

$$
\left(D_{q}^{0} h\right)(t)=h(t) \quad \text { and } \quad\left(D_{q}^{k} h\right)(t)=D_{q}\left(D_{q}^{k-1} h\right)(t), \quad k \in \mathbb{N} .
$$


The $q$-integral of a function $h$ defined on the interval $[0, b]$ is given by

$$
\left(I_{q} h\right)(t)=\int_{0}^{t} h(s) d_{q} s=t(1-q) \sum_{i=0}^{\infty} h\left(t q^{i}\right) q^{i}, \quad t \in[0, b] .
$$

If $a \in[0, b]$ and $h$ is defined in the interval $[0, b]$, then its integral from $a$ to $b$ is defined by

$$
\int_{a}^{b} h(s) d_{q} s=\int_{0}^{b} h(s) d_{q} s-\int_{0}^{a} h(s) d_{q} s .
$$

Similar to derivatives, an operator $I_{q}^{k}$ is given by

$$
\left(I_{q}^{0} h\right)(t)=h(t) \quad \text { and } \quad\left(I_{q}^{k} h\right)(t)=I_{q}\left(I_{q}^{k-1} h\right)(t), \quad k \in \mathbb{N} .
$$

The fundamental theorem of calculus applies to these operators $D_{q}$ and $I_{q}$, i.e.,

$$
\left(D_{q} I_{q} h\right)(t)=h(t)
$$

and if $h$ is continuous at $t=0$, then

$$
\left(I_{q} D_{q} h\right)(t)=h(t)-h(0) .
$$

For any $s, t>0$, the $q$-beta function is defined by

$$
B_{q}(s, t)=\int_{0}^{1} u^{(s-1)}(1-q u)^{(t-1)} d_{q} u .
$$

The expression of $q$-beta function in terms of the $q$-gamma function can be written as

$$
B_{q}(s, t)=\frac{\Gamma_{q}(s) \Gamma_{q}(t)}{\Gamma_{q}(s+t)} .
$$

Definition 2.1 Let $v \geq 0$ and $h$ be a function defined on $[0, T]$. The fractional $q$-integral of Riemann-Liouville type is given by $\left(I_{q}^{0} h\right)(t)=h(t)$ and

$$
\left(I_{q}^{v} h\right)(t)=\frac{1}{\Gamma_{q}(v)} \int_{0}^{t}(t-q s)^{(v-1)} h(s) d_{q} s, \quad v>0, t \in[0, T] .
$$

Definition 2.2 The fractional $q$-derivative of Riemann-Liouville type of order $v \geq 0$ is defined by $\left(D_{q}^{0} h\right)(t)=h(t)$ and

$$
\left(D_{q}^{v} h\right)(t)=\left(D_{q}^{l} I_{q}^{l-v} h\right)(t), \quad v>0,
$$

where $l$ is the smallest integer greater than or equal to $v$.

Lemma 2.3 [4] Let $\alpha, \beta \geq 0$ and $f$ be a function defined in $[0, T]$. Then the following formulas hold:

(1) $\left(I_{q}^{\beta} I_{q}^{\alpha} f\right)(t)=\left(I_{q}^{\alpha+\beta} f\right)(t)$,

(2) $\left(D_{q}^{\alpha} I_{q}^{\alpha} f\right)(t)=f(t)$. 
Lemma 2.4 [6] Let $\alpha>0$ and $n$ be a positive integer. Then the following equality holds:

$$
\left(I_{q}^{\alpha} D_{q}^{n} f\right)(t)=\left(D_{q}^{n} I_{q}^{\alpha} f\right)(t)-\sum_{i=0}^{n-1} \frac{t^{\alpha-n+i}}{\Gamma_{q}(\alpha+i-n+1)}\left(D_{q}^{i} f\right)(0) .
$$

\section{New concepts of fractional quantum calculus}

Let us define a $q$-shifting operator as

$$
{ }_{a} \Phi_{q}(m)=q m+(1-q) a .
$$

For any positive integer $k$, we have

$$
{ }_{a} \Phi_{q}^{k}(m)={ }_{a} \Phi_{q}^{k-1}\left({ }_{a} \Phi_{q}(m)\right) \quad \text { and } \quad{ }_{a} \Phi_{q}^{0}(m)=m .
$$

By computing directly, we get the following results.

Property 3.1 For any $m, n \in \mathbb{R}$ and for all positive integer $k, j$, we have:

(i) ${ }_{a} \Phi_{q}^{k}(m)={ }_{a} \Phi_{q^{k}}(m)$;

(ii) ${ }_{a} \Phi_{q}^{j}\left({ }_{a} \Phi_{q}^{k}(m)\right)={ }_{a} \Phi_{q}^{k}\left({ }_{a} \Phi_{q}^{j}(m)\right)={ }_{a} \Phi_{q}^{j+k}(m)$;

(iii) ${ }_{a} \Phi_{q}(a)=a$;

(iv) ${ }_{a} \Phi_{q}^{k}(m)-a=q^{k}(m-a)$;

(v) $m-{ }_{a} \Phi_{q}^{k}(m)=\left(1-q^{k}\right)(m-a)$;

(vi) ${ }_{a} \Phi_{q}^{k}(m)=m \frac{a}{m} \Phi_{q}^{k}(1)$ for $m \neq 0$;

(vii) ${ }_{a} \Phi_{q}(m)-{ }_{a} \Phi_{q}^{k}(n)=q\left(m-{ }_{a} \Phi_{q}^{k-1}(n)\right)$.

The $q$-analog of the Pochhammer symbol is defined by

$$
(m ; q)_{0}=1, \quad(m ; q)_{k}=\prod_{i=0}^{k-1}\left(1-q^{i} m\right), \quad k \in \mathbb{N} \cup\{\infty\} .
$$

We also define the new power of $q$-shifting operator as

$$
(n-m)_{a}^{(0)}=1, \quad(n-m)_{a}^{(k)}=\prod_{i=0}^{k-1}\left(n-{ }_{a} \Phi_{q}^{i}(m)\right), \quad k \in \mathbb{N} \cup\{\infty\} .
$$

More generally, if $\gamma \in \mathbb{R}$, then

$$
(n-m)_{a}^{(\gamma)}=\prod_{i=0}^{\infty} \frac{n-{ }_{a} \Phi_{q}^{i}(m)}{n-{ }_{a} \Phi_{q}^{\gamma+i}(m)} .
$$

If $a=0$, then ${ }_{0} \Phi_{q}^{i}(m)=m q^{i}$ which implies that (3.4) and (3.5) are reduced to the classical $q$-analog of the power function $(n-m)^{k}$ in (2.2) and (2.3), respectively.

Property 3.2 For any $\gamma, n, m \in \mathbb{R}$ with $n \neq a$ and $k \in \mathbb{N} \cup\{\infty\}$, we have:

(i) $(n-m)_{a}^{(k)}=(n-a)^{k}\left(\frac{m-a}{n-a} ; q\right)_{k}$;

(ii) $(n-m)_{a}^{(\gamma)}=(n-a)^{\gamma} \prod_{i=0}^{\infty} \frac{1-\frac{m-a}{n-a} q^{i}}{1-\frac{m-a}{n-a} q^{\gamma+i}}=(n-a)^{\gamma} \frac{\left(\frac{m-a}{n-a} ; q\right)_{\infty}}{\left(\frac{m-a}{n-a} q^{\gamma} ; q\right)_{\infty}}$;

(iii) $\left(n-{ }_{a} \Phi_{q}^{k}(n)\right)_{a}^{(\gamma)}=(n-a)^{\gamma} \frac{\left(q^{k} ; q\right)_{\infty}}{\left(q^{\gamma+k} ; q\right)_{\infty}}$. 
Proof To prove (i), for any $n, m \in \mathbb{R}$ with $n \neq a$, it follows that

$$
\begin{aligned}
(n-m)_{a}^{k} & =(n-m)\left(n-{ }_{a} \Phi_{q}(m)\right) \cdots\left(n-{ }_{a} \Phi_{q}^{k-1}(m)\right) \\
& =(n-a-(m-a))\left(n-a-\left({ }_{a} \Phi_{q}(m)-a\right)\right) \cdots\left(n-a-\left({ }_{a} \Phi_{q}^{k-1}(m)-a\right)\right) \\
& =(n-a)^{k}\left(1-\frac{m-a}{n-a}\right)\left(1-\frac{m-a}{n-a} q\right) \cdots\left(1-\frac{m-a}{n-a} q^{k-1}\right) \\
& =(n-a)^{k}\left(\frac{m-a}{n-a} ; q\right) .
\end{aligned}
$$

Applying the method to prove (i) for (3.5), we have the results in (ii). Using the Pochhammer symbol, we obtain the last relation (ii) as requested.

Substituting $m={ }_{a} \Phi_{q}^{k}(n)$ in (ii) and using Property 3.1(v), we obtain the desired result in (iii).

The $q$-derivative of a function $f$ on interval $[a, b]$ is defined by

$$
\left({ }_{a} D_{q} f\right)(t)=\frac{f(t)-f\left({ }_{a} \Phi_{q}(t)\right)}{(1-q)(t-a)}, \quad t \neq a, \quad \text { and } \quad\left({ }_{a} D_{q} f\right)(a)=\lim _{t \rightarrow a}\left({ }_{a} D_{q} f\right)(t),
$$

and the $q$-derivatives of higher order are given by

$$
\left({ }_{a} D_{q}^{0} f\right)(t)=f(t) \quad \text { and } \quad\left({ }_{a} D_{q}^{k} f\right)(t)={ }_{a} D_{q}^{k-1}\left({ }_{a} D_{q} f\right)(t), \quad k \in \mathbb{N} .
$$

The $q$-derivative of a product and ratio of functions $f$ and $g$ on $[a, b]$ are

$$
\begin{aligned}
{ }_{a} D_{q}(f g)(t) & =f(t)_{a} D_{q} g(t)+g\left({ }_{a} \Phi_{q}(t)\right)_{a} D_{q} f(t) \\
& =g(t)_{a} D_{q} f(t)+f\left({ }_{a} \Phi_{q}(t)\right)_{a} D_{q} g(t),
\end{aligned}
$$

and

$$
{ }_{a} D_{q}\left(\frac{f}{g}\right)(t)=\frac{g(t)_{a} D_{q} f(t)-f(t)_{a} D_{q} g(t)}{g(t) g\left({ }_{a} \Phi_{q}(t)\right)},
$$

where $g(t) g\left({ }_{a} \Phi_{q}(t)\right) \neq 0$.

The $q$-integral of a function $f$ defined on the interval $[a, b]$ is given by

$$
\left({ }_{a} I_{q} f\right)(t)=\int_{a}^{t} f(s){ }_{a} d s=(1-q)(t-a) \sum_{i=0}^{\infty} q^{i} f\left({ }_{a} \Phi_{q^{i}}(t)\right), \quad t \in[a, b] .
$$

Also similar to the derivative, an operator ${ }_{a} I_{q}^{k}$ is given by

$$
\left({ }_{a} I_{q}^{0} f\right)(t)=f(t) \quad \text { and } \quad\left({ }_{a} I_{q}^{k} f\right)(t)={ }_{a} I_{q}^{k-1}\left({ }_{a} I_{q} f\right)(t), \quad k \in \mathbb{N} .
$$

The fundamental theorem of calculus applies to these operator ${ }_{a} D_{q}$ and ${ }_{a} I_{q}$, i.e.,

$$
\left({ }_{a} D_{q a} I_{q} f\right)(t)=f(t)
$$


and if $f$ is continuous at $t=a$, then

$$
\left({ }_{a} I_{q a} D_{q} f\right)(t)=f(t)-f(a) .
$$

The formula for $q$-integration by parts on an interval $[a, b]$ is

$$
\int_{a}^{b} f(s)_{a} D_{q} g(s)_{a} d_{q} s=\left.(f g)(t)\right|_{a} ^{b}-\int_{a}^{b} g\left({ }_{a} \Phi_{q}(s)\right)_{a} D_{q} f(s)_{a} d_{q} s .
$$

The reversing order of $q$-integration formula on $[a, b]$ is given by

$$
\int_{a}^{t} \int_{a}^{s} f(r){ }_{a} d_{q} r_{a} d_{q} s=\int_{a}^{t} \int_{a}^{t} \Phi_{q}(r)
$$

Then, from (3.15), the multiple $q$-integrals can be converted to a single $q$-integral on $[a, b]$ as

$$
\begin{aligned}
{ }_{a} I_{q}^{n} f(t) & =\int_{a}^{t} \int_{a}^{x_{n-1}} \cdots \int_{a}^{x_{1}} f(s){ }_{a} d_{q} s_{a} d_{q} x_{1} \cdots{ }_{a} d_{q} x_{n-1} \\
& =\frac{1}{\Gamma_{q}(n)} \int_{a}^{t}\left(t-{ }_{a} \Phi_{q}(s)\right)_{a}^{(n-1)} f(s){ }_{a} d_{q} s .
\end{aligned}
$$

Let us give the new definitions of Riemann-Liouville fractional $q$-integral and $q$ difference on interval $[a, b]$.

Definition 3.3 Let $v \geq 0$ and $f$ be a function defined on $[a, b]$. The fractional $q$-integral of Riemann-Liouville type is given by $\left({ }_{a} I_{q}^{0} f\right)(t)=h(t)$ and

$$
\left({ }_{a} I_{q}^{v} f\right)(t)=\frac{1}{\Gamma_{q}(v)} \int_{a}^{t}\left(t-{ }_{a} \Phi_{q}(s)\right)_{a}^{(v-1)} f(s){ }_{a} d_{q} s, \quad v>0, t \in[a, b] .
$$

Definition 3.4 The fractional $q$-derivative of Riemann-Liouville type of order $v \geq 0$ on interval $[a, b]$ is defined by $\left({ }_{a} D_{q}^{0} f\right)(t)=f(t)$ and

$$
\left({ }_{a} D_{q}^{v} f\right)(t)=\left({ }_{a} D_{q^{a}}^{l} I_{q}^{l-v} f\right)(t), \quad v>0
$$

where $l$ is the smallest integer greater than or equal to $v$.

In [21], we have the following formula for $t \in[a, b], \alpha \in \mathbb{R}$ :

$$
{ }_{a} D_{q}(t-a)^{\alpha}=[\alpha]_{q}(t-a)^{\alpha-1} .
$$

It is easy to verify that

$$
{ }_{a} D_{q}^{l}(t-a)^{\alpha}=\frac{\Gamma_{q}(\alpha+1)}{\Gamma_{q}(\alpha-l+1)}(t-a)^{\alpha-l},
$$

where $l$ is a positive integer. The next result gives the generalization of (3.20). 
Lemma 3.5 Let $v>0, \alpha \in \mathbb{R}$. Then for $t \in[a, b]$, the following relation holds:

$$
{ }_{a} D_{q}^{\nu}(t-a)^{\alpha}=\frac{\Gamma_{q}(\alpha+1)}{\Gamma_{q}(\alpha-v+1)}(t-a)^{\alpha-\nu} .
$$

Proof From Definitions 3.3-3.4, we have

$$
\begin{aligned}
{ }_{a} D_{q}^{v}(t-a)^{\alpha} & ={ }_{a} D_{q}^{l} I_{q}^{l-v}(t-a)^{\alpha} \\
& ={ }_{a} D_{q}^{l} \frac{1}{\Gamma_{q}(l-v)} \int_{a}^{t}\left(t-{ }_{a} \Phi_{q}(s)\right)_{a}^{(l-v-1)}(s-a)^{\alpha}{ }_{a} d_{q} s .
\end{aligned}
$$

Using (3.10) and applying Property 3.1(iv), Property 3.2(iii), it follows that

$$
\begin{aligned}
& \int_{a}^{t}\left(t-{ }_{a} \Phi_{q}(s)\right)_{a}^{(l-v-1)}(s-a)^{\alpha}{ }_{a} d_{q} s \\
& \left.\quad=(1-q)(t-a) \sum_{i=0}^{\infty} q^{i}\left(t-{ }_{a} \Phi_{q}^{i+1}(t)\right)_{a}^{(l-v-1)}{ }_{a} \Phi_{q}^{i}(t)-a\right)^{\alpha} \\
& \quad=(1-q)(t-a) \sum_{i=0}^{\infty} q^{i}(t-a)^{l-v-1} \frac{\left(q^{i+1} ; q\right)_{\infty}}{\left(q^{l-v+i} ; q\right)_{\infty}} \cdot q^{\alpha i}(t-a)^{\alpha} \\
& \quad=(1-q)(t-a)^{l-v+\alpha} \sum_{i=0}^{\infty} q^{i}\left(1-q^{i}\right)^{(l-v-1)} q^{\alpha i} \\
& =(t-a)^{l-v+\alpha} \int_{0}^{1}(1-q s)^{(l-v-1)} s^{(\alpha)} d_{q} s \\
& =(t-a)^{l-v+\alpha} B_{q}(l-v, \alpha+1) .
\end{aligned}
$$

Applying (3.20) for (3.22)-(3.23), we obtain the desired formula in (3.21).

It follows from (2.4), (3.10), and Properties 3.1(v) and 3.2(iii) that the Riemann-Liouville fractional $q$-integral (3.17) can be written in the form of an infinite series as

$$
\begin{aligned}
{ }_{a} I_{q}^{v} f(t) & =\frac{(1-q)(t-a)}{\Gamma_{q}(v)} \sum_{i=0}^{\infty} q^{i}\left(t-{ }_{a} \Phi_{q}^{i+1}(t)\right)_{a}^{(\nu-1)} f\left({ }_{a} \Phi_{q}^{i}(t)\right) \\
& =(1-q)^{\nu}(t-a)^{\nu} \sum_{i=0}^{\infty} q^{i} \frac{\left(q^{\nu} ; q\right)_{i}}{(q ; q)_{i}} f\left({ }_{a} \Phi_{q}^{i}(t)\right) .
\end{aligned}
$$

We recall the definition of the basic $q$-hypergeometric function as

$$
{ }_{r} F_{s}\left[c_{1}, \ldots, c_{r} ; d_{1}, \ldots, d_{s} ; x\right]=\sum_{k=0}^{\infty} \frac{\left(c_{1} ; q\right)_{k} \cdots\left(c_{r} ; q\right)_{k}}{(q ; q)_{k}\left(d_{1} ; q\right)_{k} \cdots\left(d_{s} ; q\right)_{k}} x^{k}
$$

The $q$-Vandermonde reversing the order of summation [25] is

$$
{ }_{2} F_{1}\left[q^{-n}, b ; c ; \frac{c q^{n}}{b}\right]=\frac{(c / b ; q)_{n}}{(c ; q)_{n}} .
$$


Lemma 3.6 Let $\alpha, \beta \in \mathbb{R}^{+}$, and $f$ be a continuous function on $[a, b], a \geq 0$. The RiemannLiouville fractional q-integral has the following semi-group property:

$$
{ }_{a} I_{q}^{\beta} I_{q}^{\alpha} f(t)={ }_{a} I_{q a}^{\alpha} I_{q}^{\beta} f(t)={ }_{a} I_{q}^{\alpha+\beta} f(t) .
$$

Proof By taking into account of (3.24) and using Property 3.1, we have

$$
{ }_{a} I_{q}^{\alpha}\left(I_{q}^{\beta} f(t)\right)=(1-q)^{\alpha+\beta}(t-a)^{\alpha+\beta} \sum_{i=0}^{\infty} q^{i(1+\beta)} \frac{\left(q^{\alpha} ; q\right)_{i}}{(q ; q)_{i}} \sum_{j=0}^{\infty} q^{j} \frac{\left(q^{\beta} ; q\right)_{j}}{(q ; q)_{j}} f\left({ }_{a} \Phi_{q}^{i+j}(t)\right) .
$$

Applying the substitution $k=i+j$, we obtain

$$
\begin{aligned}
{ }_{a} I_{q a}^{\alpha}\left(I_{q}^{\beta} f(t)\right)= & (1-q)^{\alpha+\beta}(t-a)^{\alpha+\beta} \\
& \times \sum_{i=0}^{\infty} q^{i(1+\beta)} \frac{\left(q^{\alpha} ; q\right)_{i}}{(q ; q)_{i}} \sum_{k=i}^{\infty} q^{k-i} \frac{\left(q^{\beta} ; q\right)_{k-i}}{(q ; q)_{k-i}} f\left({ }_{a} \Phi_{q}^{k}(t)\right) .
\end{aligned}
$$

In (3.29) we interchange the order of the summations to get

$$
{ }_{a} I_{q}^{\alpha}\left(I_{q}^{\beta} f(t)\right)=(1-q)^{\alpha+\beta}(t-a)^{\alpha+\beta} \sum_{k=0}^{\infty} q^{k} f\left({ }_{a} \Phi_{q}^{k}(t)\right) \sum_{i=0}^{k} q^{i \beta} \frac{\left(q^{\alpha} ; q\right)_{i}}{(q ; q)_{i}} \frac{\left(q^{\beta} ; q\right)_{k-i}}{(q ; q)_{k-i}} .
$$

It is easy to verify that

$$
\frac{\left(q^{\beta} ; q\right)_{k-i}}{(q ; q)_{k-i}}=\frac{\left(q^{-k} ; q\right)_{i}}{\left(q^{1-k-\beta} ; q\right)_{i}} q^{(1-\beta) i}
$$

Consequently,

$$
\begin{aligned}
{ }_{a} I_{q a}^{\alpha}\left(I_{q}^{\beta} f(t)\right)= & (1-q)^{\alpha+\beta}(t-a)^{\alpha+\beta} \\
& \times \sum_{k=0}^{\infty} q^{k} f\left({ }_{a} \Phi_{q}^{k}(t)\right) \frac{\left(q^{\beta} ; q\right)_{k}}{(q ; q)_{k}}{ }_{2} F_{1}\left(q^{-k}, q^{\alpha} ; q^{1-k-\beta} ; q\right) .
\end{aligned}
$$

From (3.26), we have

$$
{ }_{2} F_{1}\left(q^{-k}, q^{\alpha} ; q^{1-k-\beta} ; q\right)=\frac{\left(q^{1-k-\beta-\alpha} ; q\right)_{k}}{\left(q^{1-k-\beta} ; q\right)_{k}} q^{k \alpha}=\frac{\left(q^{\alpha+\beta} ; q\right)_{k}}{\left(q^{\beta} ; q\right)_{k}} .
$$

Substituting (3.32) into (3.31), we obtain the series representation of ${ }_{a} I_{q}^{\alpha+\beta} f(t)$ and (3.27) holds.

Lemma 3.7 Let $f$ be a q-integrable function on $[a, b]$. Then the following equality holds:

$$
{ }_{a} D_{q}^{\alpha} I_{q}^{\alpha} f(t)=f(t), \quad \text { for } \alpha>0, t \in[a, b] .
$$

Proof If $\alpha=n, n \in \mathbb{N}$, then ${ }_{a} D_{q a}^{n} I_{q}^{n} f(t)=f(t)$. For a positive noninteger $\alpha, n-1<\alpha<n$, $n \in \mathbb{N}$, by using Lemma 3.6, we obtain

$$
{ }_{a} D_{q}^{\alpha} I_{q}^{\alpha} f(t)={ }_{a} D_{q}^{n} a_{q}^{n-\alpha}{ }_{a} I_{q}^{\alpha} f(t)={ }_{a} D_{q}^{n} I_{q}^{n} f(t)=f(t),
$$

for all $t \in[a, b]$. 
Lemma 3.8 For any $t, s \in[a, b]$. The following formulas hold:

(i) ${ }_{a}^{t} D_{q}(t-s)_{a}^{(\alpha)}=[\alpha]_{q}(t-s)_{a}^{(\alpha-1)}$;

(ii) ${ }_{a}^{s} D_{q}(t-s)_{a}^{(\alpha)}=-[\alpha]_{q}\left(t-{ }_{a} \Phi_{q}(s)\right)_{a}^{(\alpha-1)}$,

where ${ }_{a}^{i} D_{q}$ denotes the $q$-derivative with respect to variable $i$.

Proof From (3.6) and Property 3.1(vii), we have

$$
\begin{aligned}
& { }_{a}^{t} D_{q}(t-s)_{a}^{(\alpha)}=\frac{(t-s)_{a}^{(\alpha)}-\left({ }_{a} \Phi_{q}(t)-s\right)_{a}^{(\alpha)}}{(1-q)(t-a)}
\end{aligned}
$$

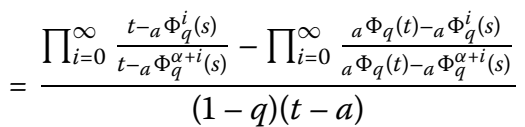

$$
\begin{aligned}
& =\frac{(t-s)_{a}^{(\alpha-1)}\left[t-{ }_{a} \Phi_{q}^{\alpha-1}(s)-q^{\alpha-1}\left({ }_{a} \Phi_{q}(t)-s\right)\right]}{(1-q)(t-a)} \\
& =[\alpha]_{q}(t-s)_{a}^{(\alpha-1)} \text {. }
\end{aligned}
$$

To prove (ii), we use (3.6) with respect to $s$ and Property 3.1(v). We omit the details.

Lemma 3.9 Let $\alpha>0$ and $p$ be a positive integer. Then for $t \in[a, b]$ the following equality holds:

$$
{ }_{a} I_{q a}^{\alpha} D_{q}^{p} f(t)={ }_{a} D_{q}^{p} I_{q}^{\alpha} f(t)-\sum_{k=0}^{p-1} \frac{(t-a)^{\alpha-p+k}}{\Gamma_{q}(\alpha+k-p+1)}{ }_{a} D_{q}^{k} f(a) .
$$

Proof Let $\alpha$ be a positive constant. Now, we will prove the formula (3.34) by using the mathematical induction. Suppose that $p=1$. By Lemma 3.8, we get

$$
{ }_{a}^{s} D_{q}\left[(t-s)_{a}^{(\alpha-1)} f(s)\right]=\left(t-{ }_{a} \Phi_{q}(s)\right)_{a}^{(\alpha-1)}{ }_{a} D_{q} f(s)-[\alpha-1]_{q}\left(t-{ }_{a} \Phi_{q}(s)\right)_{a}^{(\alpha-2)} f(s) .
$$

Therefore, by Lemma 3.8 and Property 3.1(iii), we obtain

$$
\begin{aligned}
{ }_{a} I_{q a}^{\alpha} D_{q} f(t) & =\frac{1}{\Gamma_{q}(\alpha)} \int_{a}^{t}\left(t-{ }_{a} \Phi_{q}(s)\right)_{a}^{(\alpha-1)}{ }_{a} D_{q} f(s)_{a} d s \\
& =\frac{[\alpha-1]_{q}}{\Gamma_{q}(\alpha)} \int_{a}^{t}\left(t-{ }_{a} \Phi_{q}(s)\right)_{a}^{(\alpha-2)} f(s)_{a} d s+\frac{1}{\Gamma_{q}(\alpha)}\left[(t-s)_{a}^{(\alpha-1)} f(s)\right]_{s=a}^{s=t} \\
& ={ }_{a} D_{q a} I_{q}^{\alpha} f(t)-\frac{(t-a)^{\alpha-1}}{\Gamma_{q}(\alpha)} f(a) .
\end{aligned}
$$

Next, suppose that (3.34) holds for $p \in \mathbb{N}$. Then we have

$$
\begin{aligned}
{ }_{a} I_{q a}^{\alpha} D_{q}^{p+1} f(t) & ={ }_{a} I_{q a}^{\alpha} D_{q a}^{p} D_{q} f(t) \\
& ={ }_{a} D_{q}^{p} a_{q a}^{\alpha} D_{q} f(t)-\sum_{k=0}^{p-1} \frac{(t-a)^{\alpha-p+k}}{\Gamma_{q}(\alpha+k-p+1)}{ }_{a} D_{q}^{k+1} f(a) \\
& ={ }_{a} D_{q}^{p}\left[{ }_{a} D_{q a} I_{q}^{\alpha} f(t)-\frac{(t-a)^{\alpha-1}}{\Gamma_{q}(\alpha)} f(a)\right]-\sum_{k=0}^{p-1} \frac{(t-a)^{\alpha-p+k}}{\Gamma_{q}(\alpha+k-p+1)}{ }_{a} D_{q}^{k+1} f(a)
\end{aligned}
$$




$$
\begin{aligned}
& ={ }_{a} D_{q}^{p+1}{ }_{a} I_{q}^{\alpha} f(t)-\frac{(t-a)^{\alpha-1-p}}{\Gamma_{q}(\alpha-p)} f(a)-\sum_{k=1}^{p} \frac{(t-a)^{\alpha-(p+1)+k}}{\Gamma_{q}(\alpha+k-(p+1)+1)}{ }_{a} D_{q}^{k} f(a) \\
& ={ }_{a} D_{q}^{p+1}{ }_{a} I_{q}^{\alpha} f(t)-\sum_{k=0}^{p} \frac{(t-a)^{\alpha-(p+1)+k}}{\Gamma_{q}(\alpha+k-(p+1)+1)}{ }_{a} D_{q}^{k} f(a) .
\end{aligned}
$$

\section{Impulsive fractional $q$-difference equations}

Let $J=[0, T], J_{0}=\left[t_{0}, t_{1}\right], J_{k}=\left(t_{k}, t_{k+1}\right]$ for $k=1,2,3, \ldots, m$. Let $P C(J, \mathbb{R})=\{x: J \rightarrow \mathbb{R}$, $x(t)$ is continuous everywhere except for some $t_{k}$ at which $x\left(t_{k}^{+}\right)$and $x\left(t_{k}^{-}\right)$exist and $\left.x\left(t_{k}^{-}\right)=x\left(t_{k}\right), k=1,2,3, \ldots, m\right\}$. For $\gamma \in \mathbb{R}_{+}$, we introduce the space $C_{\gamma, k}\left(J_{k}, \mathbb{R}\right)=\left\{x: J_{k} \rightarrow\right.$ $\left.\mathbb{R}:\left(t-t_{k}\right)^{\gamma} x(t) \in C\left(J_{k}, \mathbb{R}\right)\right\}$ with the norm $\|x\|_{C_{\gamma, k}}=\sup _{t \in J_{k}}\left|\left(t-t_{k}\right)^{\gamma} x(t)\right|$ and $P C_{\gamma}=\{x: J \rightarrow$ $\mathbb{R}:$ for each $t \in J_{k}$ and $\left.\left(t-t_{k}\right)^{\gamma} x(t) \in C\left(J_{k}, \mathbb{R}\right), k=0,1,2, \ldots, m\right\}$ with the norm $\|x\|_{P C_{\gamma}}=$ $\max \left\{\sup _{t \in J_{k}}\left|\left(t-t_{k}\right)^{\gamma} x(t)\right|: k=0,1,2, \ldots, m\right\}$. Clearly $P C_{\gamma}$ is a Banach space.

\subsection{Impulsive fractional $q$-difference equation of order $0<\alpha \leq 1$}

In this subsection, we initiate the study of the existence and uniqueness of solutions for the following initial value problem for impulsive fractional $q$-difference equation of order $0<\alpha \leq 1$

$$
\left\{\begin{array}{l}
t_{k} D_{q_{k}}^{\alpha} x(t)=f(t, x(t)), \quad t \in J, t \neq t_{k}, \\
\widetilde{\Delta} x\left(t_{k}\right)=\varphi_{k}\left(x\left(t_{k}\right)\right), \quad k=1,2, \ldots, m, \\
x(0)=0,
\end{array}\right.
$$

where $t_{k} D_{q_{k}}^{\alpha}$ is the Riemann-Liouville fractional $q$-difference of order $\alpha$ defined by (3.18) on interval $J_{k}, 0<q_{k}<1$ for $k=0,1,2, \ldots, m, 0=t_{0}<t_{1}<t_{2}<\cdots<t_{k}<\cdots<t_{m}<t_{m+1}=T$, $f: J \times \mathbb{R} \rightarrow \mathbb{R}$ is a continuous function, $\varphi_{k} \in C(\mathbb{R}, \mathbb{R})$. The notation $\widetilde{\Delta} x\left(t_{k}\right)$ is defined by

$$
\widetilde{\Delta} x\left(t_{k}\right)={ }_{t_{k}} I_{q_{k}}^{1-\alpha} x\left(t_{k}^{+}\right)-{ }_{t_{k-1}} I_{q_{k-1}}^{1-\alpha} x\left(t_{k}\right), \quad k=1,2, \ldots, m,
$$

where $t_{k} I_{q_{k}}^{1-\alpha}$ is the Riemann-Liouville fractional $q$-integral of order $1-\alpha$ defined by (3.17) on $J_{k}$. It should be noticed that if $\alpha=1$ in (4.2), then $\widetilde{\Delta} x\left(t_{k}\right)=\Delta x\left(t_{k}\right)=x\left(t_{k}^{+}\right)-x\left(t_{k}\right)$ for $k=1,2, \ldots, m$.

Lemma 4.1 If $x \in P C(J, \mathbb{R})$ is a solution of (4.1), then for any $t \in J_{k}, k=0,1,2, \ldots, m$,

$$
x(t)=\frac{\left(t-t_{k}\right)^{\alpha-1}}{\Gamma_{q_{k}}(\alpha)}\left[\sum_{0<t_{k}<t}\left(t_{k-1} I_{q_{k-1}}^{1} f\left(t_{k}, x\left(t_{k}\right)\right)+\varphi_{k}\left(x\left(t_{k}\right)\right)\right)\right]+{ }_{t_{k}} I_{q_{k}}^{\alpha} f(t, x(t)),
$$

with $\sum_{0<0}(\cdot)=0$. The converse is also true.

Proof In view of Definition 3.4, for $t \in J_{0}$ and $t_{0}=0$, it follows that

$$
{ }_{0} I_{q_{0} 0}^{\alpha} D_{q_{0}}^{\alpha} x(t)={ }_{0} I_{q_{0} 0}^{\alpha} D_{q_{0} 0} I_{q_{0}}^{1-\alpha} x(t)={ }_{0} I_{q_{0}}^{\alpha} f(t, x(t))
$$

By Lemmas 3.7 and 3.9, $t \in J_{0}$, we have

$$
x(t)=c_{0} \frac{t^{\alpha-1}}{\Gamma_{q_{0}}(\alpha)}+{ }_{0} I_{q_{0}}^{\alpha} f(t, x(t))
$$


where $c_{0}={ }_{0} I_{q_{0}}^{1-\alpha} x(0)$. The initial condition $x(0)=0$ leads to $c_{0}=0$ which yields for $t \in J_{0}$,

$$
x(t)={ }_{0} I_{q_{0}}^{\alpha} f(t, x(t)) .
$$

The Riemann-Liouville fractional $q_{0}$-integrating for order $1-\alpha$ for $t=t_{1}$ leads to

$$
{ }_{0} I_{q_{0}}^{1-\alpha} x\left(t_{1}\right)={ }_{0} I_{q_{0}}^{1} f\left(t_{1}, x\left(t_{1}\right)\right)
$$

For $t \in J_{1}$, taking the Riemann-Liouville fractional $q_{1}$-integral of order $\alpha$ to (4.1) and using the above process, we have

$$
x(t)=\frac{\left(t-t_{1}\right)^{\alpha-1}}{\Gamma_{q_{1}}(\alpha)} t_{1} I_{q_{1}}^{1-\alpha} x\left(t_{1}^{+}\right)+{ }_{t_{1}} I_{q_{1}}^{\alpha} f(t, x(t))
$$

Since $t_{1} I_{q_{1}}^{1-\alpha} x\left(t_{1}^{+}\right)={ }_{0} I_{q_{0}}^{1-\alpha} x\left(t_{1}\right)+\varphi_{1}\left(x\left(t_{1}\right)\right)$, it follows using (4.4) for $t \in J_{1}$ that

$$
x(t)=\frac{\left(t-t_{1}\right)^{\alpha-1}}{\Gamma_{q_{1}}(\alpha)}\left[{ }_{0} I_{q_{0}}^{1} f\left(t_{1}, x\left(t_{1}\right)\right)+\varphi_{1}\left(x\left(t_{1}\right)\right)\right]+{ }_{t_{1}} I_{q_{1}}^{\alpha} f(t, x(t)) .
$$

By computing directly, for $t=t_{2}$, we obtain from (4.5)

$$
{ }_{t_{1}} I_{q_{1}}^{1-\alpha} x\left(t_{2}\right)={ }_{0} I_{q_{0}}^{1} f\left(t_{1}, x\left(t_{1}\right)\right)+{ }_{t_{1}} I_{q_{1}}^{1} f\left(t_{2}, x\left(t_{2}\right)\right)+\varphi_{1}\left(x\left(t_{1}\right)\right) .
$$

Applying the Riemann-Liouville fractional $q_{2}$-integrating of order $\alpha$ for (4.1) from $t_{2}$ to $t$, where $t \in J_{2}$, then we have

$$
\begin{aligned}
x(t)= & \frac{\left(t-t_{2}\right)^{\alpha-1}}{\Gamma_{q_{2}}(\alpha)}{ }_{t_{2}} I_{q_{2}}^{1-\alpha} x\left(t_{2}^{+}\right)+{ }_{t_{2}} I_{q_{2}}^{\alpha} f(t, x(t)) \\
= & \frac{\left(t-t_{2}\right)^{\alpha-1}}{\Gamma_{q_{2}}(\alpha)}\left[{ }_{0} I_{q_{0}}^{1} f\left(t_{1}, x\left(t_{1}\right)\right)+{ }_{t_{1}} I_{q_{1}}^{1} f\left(t_{2}, x\left(t_{2}\right)\right)+\varphi_{1}\left(x\left(t_{1}\right)\right)+\varphi_{2}\left(x\left(t_{2}\right)\right)\right] \\
& +{ }_{t_{2}} I_{q_{2}}^{\alpha} f(t, x(t)) .
\end{aligned}
$$

Repeating the above process, for $t \in J$, we obtain (4.3).

On the other hand, assume that $x$ is a solution of (4.1). Applying the Riemann-Liouville fractional $q_{k}$-derivative of order $\alpha$ on (4.3) for $t \in J_{k}, k=0,1,2, \ldots, m$ and using $\Gamma(0)=\infty$, it follows that

$$
t_{k} D_{q_{k}}^{\alpha} x(t)=f(t, x(t))
$$

It is easy to verify that $\widetilde{\Delta} x\left(t_{k}\right)=\varphi_{k}\left(x\left(t_{k}\right)\right), k=1,2, \ldots, m$ and $x(0)=0$. This completes the proof.

Theorem 4.2 Assume that the following assumptions hold:

$\left(\mathrm{H}_{1}\right) f: J \times \mathbb{R} \rightarrow \mathbb{R}$ is a continuous function and satisfies

$$
|f(t, x)-f(t, y)| \leq L|x-y|, \quad L>0, \forall t \in J, x, y \in \mathbb{R}
$$


$\left(\mathrm{H}_{2}\right) \varphi_{k}: \mathbb{R} \rightarrow \mathbb{R}, k=1,2, \ldots, m$, are continuous functions and satisfy

$$
\left|\varphi_{k}(x)-\varphi_{k}(y)\right| \leq M|x-y|, \quad M>0, \forall x, y \in \mathbb{R} .
$$

If

$$
\Lambda \leq \delta<1
$$

where

$$
\Lambda=\frac{T^{*}}{\Gamma^{*}}(L T+m M+L)
$$

$T^{*}=\max \left\{T^{\gamma+\alpha-1}, T^{\gamma+\alpha}\right\}, \Gamma^{*}=\min \left\{\Gamma_{q_{k}}(\alpha), \Gamma_{q_{k}}(\alpha+1), k=0,1,2, \ldots, m\right\}$, and $\gamma+\alpha>1$, then the nonlinear impulsive fractional q-difference initial value problem (4.1) has a unique solution on $J$.

Proof We define an operator $\mathcal{A}: P C(J, \mathbb{R}) \rightarrow P C(J, \mathbb{R})$ by

$$
(\mathcal{A} x)(t)=\frac{\left(t-t_{k}\right)^{\alpha-1}}{\Gamma_{q_{k}}(\alpha)}\left[\sum_{0<t_{k}<t}\left(t_{k-1} I_{q_{k-1}}^{1} f(s, x(s))\left(t_{k}\right)+\varphi_{k}\left(x\left(t_{k}\right)\right)\right)\right]+{ }_{t_{k}} I_{q_{k}}^{\alpha} f(s, x(s))(t),
$$

with $\sum_{0<0}(\cdot)=0$.

In addition, we define a ball $B_{r}=\left\{x \in P C_{\gamma}(J, \mathbb{R}):\|x\|_{P C_{\gamma}} \leq r\right\}$. To show that $\mathcal{A} x \in P C_{\gamma}$, we suppose $\tau_{1}, \tau_{2} \in J_{k}$, and then

$$
\begin{aligned}
& \left|\left(\tau_{1}-t_{k}\right)^{\gamma} \mathcal{A} x\left(\tau_{1}\right)-\left(\tau_{2}-t_{k}\right)^{\gamma} \mathcal{A} x\left(\tau_{2}\right)\right| \\
& \leq\left|\frac{\left(\tau_{1}-t_{k}\right)^{\gamma+\alpha-1}-\left(\tau_{2}-t_{k}\right)^{\gamma+\alpha-1}}{\Gamma_{q_{k}}(\alpha)}\right|\left[\sum_{j=1}^{k}\left(t_{j-1} I_{q_{j-1}}^{1}|f(s, x(s))|\left(t_{j}\right)+\left|\varphi_{j}\left(x\left(t_{j}\right)\right)\right|\right)\right] \\
& \quad+\left|\left(\tau_{1}-t_{k}\right)^{\gamma}{ }_{t_{k}} I_{q_{k}}^{\alpha} f(s, x(s))\left(\tau_{1}\right)-\left(\tau_{2}-t_{k}\right)^{\gamma}{ }_{t_{k}} I_{q_{k}}^{\alpha} f(s, x(s))\left(\tau_{2}\right)\right| .
\end{aligned}
$$

As $\tau_{1} \rightarrow \tau_{2}$, we have $\left|\left(\tau_{1}-t_{k}\right)^{\gamma} \mathcal{A} x\left(\tau_{1}\right)-\left(\tau_{2}-t_{k}\right)^{\gamma} \mathcal{A} x\left(\tau_{2}\right)\right| \rightarrow 0$ for each $k=0,1,2, \ldots, m$. Therefore, we get $\mathcal{A} x(t) \in P C_{\gamma}$. Now, we will show that $\mathcal{A} B_{r} \subset B_{r}$. Assume that $\sup _{t \in J}|f(t, 0)|=N_{1}$ and $\max \left\{\left|I_{k}(0)\right|: k=1,2, \ldots, m\right\}=N_{2}$, and setting

$$
\Omega=\frac{T^{*}}{\Gamma^{*}}\left(N_{1} T+m N_{2}+N_{1}\right)
$$

we choose a constant $r$ such that

$$
r \geq \frac{1}{1-\varepsilon} \Omega,
$$

where $\delta \leq \varepsilon<1$. For any $x \in B_{r}$ and for each $t \in J_{k}$, we have

$$
\begin{aligned}
|(\mathcal{A} x)(t)| \leq & \frac{\left(t-t_{k}\right)^{\alpha-1}}{\Gamma_{q_{k}}(\alpha)}\left[\sum_{0<t_{k}<t}\left(t_{k-1} I_{q_{k-1}}^{1}|f(s, x(s))|\left(t_{k}\right)+\left|\varphi_{k}\left(x\left(t_{k}\right)\right)\right|\right)\right] \\
& +t_{k} I_{q_{k}}^{\alpha}|f(s, x(s))|(t)
\end{aligned}
$$




$$
\begin{aligned}
\leq & \frac{\left(t-t_{k}\right)^{\alpha-1}}{\Gamma_{q_{k}}(\alpha)}\left[\sum _ { j = 1 } ^ { k } \left(t_{j-1} I_{q_{j-1}}^{1}(|f(s, x(s))-f(s, 0)|+|f(s, 0)|)\left(t_{j}\right)\right.\right. \\
& \left.\left.+\left(\left|\varphi_{j}\left(x\left(t_{j}\right)\right)-\varphi_{j}(0)\right|+\left|\varphi_{j}(0)\right|\right)\right)\right]+{ }_{t_{k}} I_{q_{k}}^{\alpha}(|f(s, x(s))-f(s, 0)|+|f(s, 0)|)(t) \\
\leq & \frac{\left(t-t_{k}\right)^{\alpha-1}}{\Gamma_{q_{k}}(\alpha)}\left[\left(L r+N_{1}\right) t_{k}+\left(M r+N_{2}\right) k\right]+\frac{\left(t-t_{k}\right)^{\alpha}}{\Gamma_{q_{k}}(\alpha+1)}\left(L r+N_{1}\right) .
\end{aligned}
$$

Multiplying both sides of the above inequality by $\left(t-t_{k}\right)^{\gamma}$ for each $t \in J_{k}$, we have

$$
\begin{aligned}
\left(t-t_{k}\right)^{\gamma}|(\mathcal{A} x)(t)| & \leq \frac{\left(t-t_{k}\right)^{\gamma+\alpha-1}}{\Gamma_{q_{k}}(\alpha)}\left[\left(L r+N_{1}\right) t_{k}+\left(M r+N_{2}\right) k\right]+\frac{\left(t-t_{k}\right)^{\gamma+\alpha}}{\Gamma_{q_{k}}(\alpha+1)}\left(L r+N_{1}\right) \\
& \leq \frac{T^{*}}{\Gamma^{*}}\left[\left(L r+N_{1}\right) T+\left(M r+N_{2}\right) m\right]+\frac{T^{*}}{\Gamma^{*}}\left(L r+N_{1}\right) \\
& \leq(\delta+1-\varepsilon) r \leq r .
\end{aligned}
$$

This means that $\|\mathcal{A} x\|_{P C_{\gamma}} \leq r$, which leads to $\mathcal{A} B_{r} \subset B_{r}$.

For $x, y \in P C_{\gamma}(J, \mathbb{R})$ and for each $t \in J$, we have

$$
\begin{aligned}
|(\mathcal{A} x)(t)-(\mathcal{A} y)(t)| \leq & \frac{\left(t-t_{k}\right)^{\alpha-1}}{\Gamma_{q_{k}}(\alpha)}\left[\sum _ { 0 < t _ { k } < t } \left(t_{k-1} I_{q_{k-1}}^{1}(|f(s, x(s))-f(s, y(s))|)\left(t_{k}\right)\right.\right. \\
& \left.\left.+\left|\varphi_{k}\left(x\left(t_{k}\right)\right)-\varphi_{k}\left(y\left(t_{k}\right)\right)\right|\right)\right]+t_{k} I_{q_{k}}^{\alpha}(|f(s, x(s))-f(s, y(s))|)(t) \\
\leq & \frac{\left(t-t_{k}\right)^{\alpha-1}}{\Gamma_{q_{k}}(\alpha)}\left[\sum_{j=1}^{k}\left(t_{j-1} I_{q_{j-1}}^{1}(L|x(s)-y(s)|)\left(t_{j}\right)+M\left|x\left(t_{j}\right)-y\left(t_{j}\right)\right|\right)\right] \\
& +t_{t_{k}} I_{q_{k}}^{\alpha}(L|x(s)-y(s)|)(t) \\
\leq & \frac{\left(t-t_{k}\right)^{\alpha-1}}{\Gamma_{q_{k}}(\alpha)}\left[\sum_{j=1}^{k}\left(L\left(t_{j}-t_{j-1}\right)\|x-y\|_{P C_{\gamma}}+M\|x-y\|_{P C_{\gamma}}\right)\right] \\
& +\frac{\left(t-t_{k}\right)^{\alpha}}{\Gamma_{q_{k}}(\alpha+1)} L\|x-y\|_{P C_{\gamma}} .
\end{aligned}
$$

Multiplying both sides of the above inequality by $\left(t-t_{k}\right)^{\gamma}$ for each $t \in J_{k}$, we have

$$
\begin{aligned}
\left|\left(t-t_{k}\right)^{\gamma}(\mathcal{A} x)(t)-\left(t-t_{k}\right)^{\gamma}(\mathcal{A} y)(t)\right| \leq & \frac{\left(t-t_{k}\right)^{\gamma+\alpha-1}}{\Gamma_{q_{k}}(\alpha)}\left(t_{k} L\|x-y\|_{P C_{\gamma}}+k M\|x-y\|_{P C_{\gamma}}\right) \\
& +\frac{\left(t-t_{k}\right)^{\gamma+\alpha}}{\Gamma_{q_{k}}(\alpha+1)} L\|x-y\|_{P C_{\gamma}} \\
\leq & \frac{T^{*}}{\Gamma^{*}}(L T+m M+L)\|x-y\|_{P C_{\gamma}} .
\end{aligned}
$$

It follows that

$$
\|\mathcal{A} x-\mathcal{A} y\|_{P C_{\gamma}} \leq \Lambda\|x-y\|_{P C_{\gamma}} .
$$

As $\Lambda<1$, by the Banach contraction mapping principle, $\mathcal{A}$ is a contraction. Therefore, $\mathcal{A}$ has a fixed point which is a unique solution of (4.1) on $J$. 
Example 4.3 Consider the following impulsive fractional $q$-difference initial value problem:

$$
\left\{\begin{array}{l}
t_{k} D_{\left(\frac{k^{2}+2 k+3}{k^{2}+3 k+4}\right)}^{\frac{1}{2}} x(t)=\frac{(t+1)|x(t)|}{2^{t+1}\left(t^{2}+\sqrt{6}\right)^{2}(1+|x(t)|)}+\frac{1}{2}, \quad t \in\left[0, \frac{9}{10}\right], t \neq t_{k}, \\
\widetilde{\Delta} x\left(t_{k}\right)=\frac{\left|x\left(t_{k}\right)\right|}{4(k+4)+\left|x\left(t_{k}\right)\right|}, \quad k=1,2, \ldots, 8, t_{k}=\frac{k}{10} \\
x(0)=0,
\end{array}\right.
$$

Here $\alpha=1 / 2, q_{k}=\left(k^{2}+2 k+3\right) /\left(k^{2}+3 k+4\right), k=0,1,2, \ldots, 8, m=8, T=9 / 10, f(t, x)=$ $\left(((t+1)|x|) /\left(2^{t+1}\left(t^{2}+\sqrt{6}\right)^{2}(1+|x|)\right)\right)+(1 / 2)$, and $\varphi_{k}(x)=(|x| /(4(k+4)+|x|))$. Since $\mid f(t, x)-$ $f(t, y)|\leq(19 / 120)| x-y \mid$ and $\left|\varphi_{k}(x)-\varphi_{k}(y)\right| \leq(1 / 20)|x-y|,\left(\mathrm{H}_{1}\right),\left(\mathrm{H}_{2}\right)$ are satisfied with $L=(19 / 120), M=(1 / 20)$. Choosing $\gamma=4 / 5$ and using the Maple program, we can find that $T^{*}=0.9688861612, \Gamma^{*}=0.8918490635$, and

$$
\Lambda=\frac{T^{*}}{\Gamma^{*}}(L T+m M+L) \approx 0.7613706689<1 .
$$

Hence, by Theorem 4.2, the initial value problem (4.6) has a unique solution on $[0,9 / 10]$.

\subsection{Impulsive fractional $q$-difference equation of order $1<\alpha \leq 2$}

In this subsection, we investigate the initial value problem of impulsive fractional $q$ difference equation of order $1<\alpha \leq 2$ the form

$$
\left\{\begin{array}{l}
t_{k} D_{q_{k}}^{\alpha} x(t)=f(t, x(t)), \quad t \in J, t \neq t_{k} \\
\widetilde{\Delta} x\left(t_{k}\right)=\varphi_{k}\left(x\left(t_{k}\right)\right), \quad k=1,2, \ldots, m \\
\Delta^{*} x\left(t_{k}\right)=\varphi_{k}^{*}\left(x\left(t_{k}\right)\right), \quad k=1,2, \ldots, m \\
x(0)=0, \quad{ }_{0} D_{q_{0}}^{\alpha-1} x(0)=\beta
\end{array}\right.
$$

where $\beta \in \mathbb{R}, 0=t_{0}<t_{1}<t_{2}<\cdots<t_{k}<\cdots<t_{m}<t_{m+1}=T, f: J \times \mathbb{R} \rightarrow \mathbb{R}$ is a continuous function, $\varphi_{k}, \varphi_{k}^{*} \in C(\mathbb{R}, \mathbb{R})$ for $k=1,2, \ldots, m$ and $0<q_{k}<1$ for $k=0,1,2, \ldots, m$. The notation $\widetilde{\Delta} x\left(t_{k}\right)$ is defined by (4.2) and $\Delta^{*} x\left(t_{k}\right)$ is defined by

$$
\Delta^{*} x\left(t_{k}\right)=t_{k} I_{q_{k}}^{2-\alpha} x\left(t_{k}^{+}\right)-t_{k-1} I_{q_{k-1}}^{2-\alpha} x\left(t_{k}\right), \quad k=1,2, \ldots, m,
$$

where $t_{k} I_{q_{k}}^{2-\alpha}$ is the Riemann-Liouville fractional $q$-integral of order $2-\alpha$ defined by (3.17) on $J_{k}$. It should be noticed that if $\alpha=2$, then $\widetilde{\Delta} x\left(t_{k}\right)={ }_{t_{k}} D_{q_{k}} x\left(t_{k}^{+}\right)-t_{t_{k-1}} D_{q_{k-1}} x\left(t_{k}\right)$ and $\Delta^{*} x\left(t_{k}\right)=\Delta x\left(t_{k}\right)=x\left(t_{k}^{+}\right)-x\left(t_{k}\right)$ for $k=1,2, \ldots, m$.

Lemma 4.4 The unique solution of problem (4.7) is given by

$$
\begin{aligned}
x(t)= & \frac{\left(t-t_{k}\right)^{\alpha-2}}{\Gamma_{q_{k}}(\alpha-1)}\left[\beta t_{k}+\sum_{0<t_{k}<t} \sum_{0<t_{j}<t_{k}}\left(t_{k}-t_{k-1}\right)\left(t_{j-1} I_{q_{j-1}}^{1} f\left(t_{j}, x\left(t_{j}\right)\right)+\varphi_{j}\left(x\left(t_{j}\right)\right)\right)\right. \\
& \left.+\sum_{0<t_{k}<t}\left(t_{k-1} I_{q_{k-1}}^{2} f\left(t_{k}, x\left(t_{k}\right)\right)+\varphi_{k}^{*}\left(x\left(t_{k}\right)\right)\right)\right] \\
& +\frac{\left(t-t_{k}\right)^{\alpha-1}}{\Gamma_{q_{k}}(\alpha)}\left[\beta+\sum_{0<t_{k}<t} t_{k-1} I_{q_{k-1}}^{1} f\left(t_{k}, x\left(t_{k}\right)\right)+\varphi_{k}\left(x\left(t_{k}\right)\right)\right]+{ }_{t_{k}} I_{q_{k}}^{\alpha} f(t, x(t)),
\end{aligned}
$$

with $\sum_{0<0}(\cdot)=0$. 
Proof For $t \in J_{0}$, taking the Riemann-Liouville fractional $q_{0}$-integral of order $\alpha$ for the first equation of (4.7) and using Definition 3.4 with Lemma 3.9, we get

$$
x(t)=\frac{t^{\alpha-2}}{\Gamma_{q_{0}}(\alpha-1)} C_{0}+\frac{t^{\alpha-1}}{\Gamma_{q_{0}}(\alpha)} C_{1}+{ }_{0} I_{q_{0}}^{\alpha} f(t, x(t)),
$$

where $C_{0}={ }_{0} I_{q_{0}}^{2-\alpha} x(0)$ and $C_{1}={ }_{0} I_{q_{0}}^{1-\alpha} x(0)$. The first initial condition of (4.7) implies that $C_{0}=0$.

Taking the Riemann-Liouville fractional $q_{0}$-derivative of order $\alpha-1$ for (4.10) on $J_{0}$, we have

$$
{ }_{0} D_{q_{0}}^{\alpha-1} x(t)=C_{1}+{ }_{0} I_{q_{0}}^{1} f(t, x(t)) .
$$

The second initial condition of (4.7) with (4.11) yields $C_{1}=\beta$. Therefore, (4.10) can be written as

$$
x(t)=\frac{\beta t^{\alpha-1}}{\Gamma_{q_{0}}(\alpha)}+{ }_{0} I_{q_{0}}^{\alpha} f(t, x(t))
$$

Applying the Riemann-Liouville fractional $q_{0}$-derivative of orders $1-\alpha$ and $2-\alpha$ for (4.12) at $t=t_{1}$, we have

$$
{ }_{0} I_{q_{0}}^{1-\alpha} x\left(t_{1}\right)=\beta+{ }_{0} I_{q_{0}}^{1} f\left(t_{1}, x\left(t_{1}\right)\right) \quad \text { and } \quad{ }_{0} I_{q_{0}}^{2-\alpha} x\left(t_{1}\right)=\beta t_{1}+{ }_{0} I_{q_{0}}^{2} f\left(t_{1}, x\left(t_{1}\right)\right)
$$

For $t \in J_{1}=\left(t_{1}, t_{2}\right]$, Riemann-Liouville fractional $q_{1}$-integrating (4.7), we obtain

$$
x(t)=\frac{\left(t-t_{1}\right)^{\alpha-2}}{\Gamma_{q_{1}}(\alpha-1)} t_{1} I_{q_{1}}^{2-\alpha} x\left(t_{1}^{+}\right)+\frac{\left(t-t_{1}\right)^{\alpha-1}}{\Gamma_{q_{1}}(\alpha)} t_{1} I_{q_{1}}^{1-\alpha} x\left(t_{1}^{+}\right)+{ }_{t_{1}} I_{q 1}^{\alpha} f(t, x(t)) .
$$

Using the jump conditions of (4.7) with (4.13)-(4.14) for $t \in J_{1}$, we get

$$
\begin{aligned}
x(t)= & \frac{\left(t-t_{1}\right)^{\alpha-2}}{\Gamma_{q_{1}}(\alpha-1)}\left[\beta t_{1}+{ }_{0} I_{q_{0}}^{2} f\left(t_{1}, x\left(t_{1}\right)\right)+\varphi_{1}^{*}\left(x\left(t_{1}\right)\right)\right] \\
& +\frac{\left(t-t_{1}\right)^{\alpha-1}}{\Gamma_{q_{1}}(\alpha)}\left[\beta+{ }_{0} I_{q_{0}}^{1} f\left(t_{1}, x\left(t_{1}\right)\right)+\varphi_{1}\left(x\left(t_{1}\right)\right)\right]+{ }_{t_{1}} I_{q}^{\alpha} f(t, x(t)) .
\end{aligned}
$$

Repeating the above process, for $t \in J$, we obtain (4.9) as required.

Next, we prove the existence and uniqueness of a solution to the initial value problem (4.7). We shall use the Banach fixed point theorem to accomplish this.

For convenience, we set the constants

$$
\begin{aligned}
\Psi_{1}= & \frac{\widetilde{T}}{\widetilde{\Gamma}}\left[m\left(M+M^{*}\right)+L\left(t_{m}+1\right)+L \sum_{j=1}^{m}\left(t_{j}-t_{j-1}\right) t_{j-1}\right. \\
& \left.+L \sum_{j=1}^{m} \frac{\left(t_{j}-t_{j-1}\right)^{2}}{1+q_{j-1}}+M \sum_{j=1}^{m}\left(t_{j}-t_{j-1}\right)(j-1)\right]
\end{aligned}
$$




$$
\begin{aligned}
\Psi_{2}= & \frac{\widetilde{T}}{\widetilde{\Gamma}}\left[\left(|\beta|+\Omega_{1}\right)\left(t_{m}+1\right)+m\left(\Omega_{2}+\Omega_{3}\right)+\Omega_{1} \sum_{j=1}^{m}\left(t_{j}-t_{j-1}\right) t_{j-1}\right. \\
& \left.+\Omega_{1} \sum_{j=1}^{m} \frac{\left(t_{j}-t_{j-1}\right)^{2}}{1+q_{j-1}}+\Omega_{2} \sum_{j=1}^{m}\left(t_{j}-t_{j-1}\right)(j-1)\right]
\end{aligned}
$$

where $\widetilde{T}=\max \left\{T^{\gamma+\alpha-2}, T^{\gamma+\alpha-1}, T^{\gamma+\alpha}\right\}, \widetilde{\Gamma}=\min \left\{\Gamma_{q_{k}}(\alpha-1), \Gamma_{q_{k}}(\alpha), \Gamma_{q_{k}}(\alpha+1), k=0,1,2\right.$, $\ldots, m\}$, and $\gamma+\alpha>2$.

Theorem 4.5 Assume that $\left(\mathrm{H}_{1}\right)$ and $\left(\mathrm{H}_{2}\right)$ hold. In addition we suppose that:

$\left(\mathrm{H}_{3}\right) \varphi_{k}^{*}: \mathbb{R} \rightarrow \mathbb{R}, k=1,2, \ldots, m$, are continuous functions and satisfy

$$
\left|\varphi_{k}^{*}(x)-\varphi_{k}^{*}(y)\right| \leq M^{*}|x-y|, \quad M^{*}>0, \forall x, y \in \mathbb{R}
$$

If

$$
\Psi_{1} \leq \delta<1,
$$

where $\Psi_{1}$ is defined by (4.15), then the initial value problem (4.7) has a unique solution on $J$.

Proof Firstly, in view of Lemma 4.4, we define an operator $\mathcal{Q}: P C(J, \mathbb{R}) \rightarrow P C(J, \mathbb{R})$ as

$$
\begin{aligned}
(\mathcal{Q} x)(t)= & \frac{\left(t-t_{k}\right)^{\alpha-2}}{\Gamma_{q_{k}}(\alpha-1)}\left[\beta t_{k}+\sum_{0<t_{k}<t} \sum_{0<t_{j}<t_{k}}\left(t_{k}-t_{k-1}\right)\left(t_{j-1} I_{q_{j-1}}^{1} f\left(t_{j}, x\left(t_{j}\right)\right)+\varphi_{j}\left(x\left(t_{j}\right)\right)\right)\right. \\
& \left.+\sum_{0<t_{k}<t}\left(t_{k-1} I_{q_{k-1}}^{2} f\left(t_{k}, x\left(t_{k}\right)\right)+\varphi_{k}^{*}\left(x\left(t_{k}\right)\right)\right)\right] \\
& +\frac{\left(t-t_{k}\right)^{\alpha-1}}{\Gamma_{q_{k}}(\alpha)}\left[\beta+\sum_{0<t_{k}<t}\left(t_{k-1} I_{q_{k-1}}^{1} f\left(t_{k}, x\left(t_{k}\right)\right)+\varphi_{k}\left(x\left(t_{k}\right)\right)\right)\right]+{ }_{t_{k}} I_{q_{k}}^{\alpha} f(t, x(t)),
\end{aligned}
$$

with $\sum_{0<0}(\cdot)=0$.

It is straightforward to show that $\mathcal{Q} x \in P C_{\gamma}(J, \mathbb{R})$; see Theorem 4.2. Setting $\sup _{t \in J}|f(t, 0)|=\Omega_{1}, \max \left\{\varphi_{k}(0): k=1,2, \ldots, m\right\}=\Omega_{2}$, and $\max \left\{\varphi_{k}^{*}(0): k=1,2, \ldots, m\right\}=\Omega_{3}$, we will show that $\mathcal{Q} B_{R} \subset B_{R}$, where $B_{R}=\left\{x \in P C_{\gamma}(J, \mathbb{R}):\|x\|_{P C_{\gamma}} \leq R\right\}$ and a constant $R$ satisfies

$$
R \geq \frac{\Psi_{2}}{1-\varepsilon}
$$

where $\Psi_{2}$ is defined by (4.16) and $\delta \leq \varepsilon<1$. Let $x \in B_{R}$. For each $t \in J_{k}, k=0,1,2, \ldots, m$, we have

$$
\begin{aligned}
|(\mathcal{Q} x)(t)| \leq & \frac{\left(t-t_{k}\right)^{\alpha-2}}{\Gamma_{q_{k}}(\alpha-1)}\left[|\beta| t_{k}+\sum_{0<t_{k}<t} \sum_{0<t_{j}<t_{k}}\left(t_{k}-t_{k-1}\right)\left(t_{j-1} I_{q_{j-1}}^{1}\left|f\left(t_{j}, x\left(t_{j}\right)\right)\right|+\left|\varphi_{j}\left(x\left(t_{j}\right)\right)\right|\right)\right. \\
& \left.+\sum_{0<t_{k}<t}\left(t_{k-1} I_{q_{k-1}}^{2}\left|f\left(t_{k}, x\left(t_{k}\right)\right)\right|+\left|\varphi_{k}^{*}\left(x\left(t_{k}\right)\right)\right|\right)\right]
\end{aligned}
$$




$$
\begin{aligned}
& +\frac{\left(t-t_{k}\right)^{\alpha-1}}{\Gamma_{q_{k}}(\alpha)}\left[|\beta|+\sum_{0<t_{k}<t}\left(t_{k-1} I_{q_{k-1}}^{1}\left|f\left(t_{k}, x\left(t_{k}\right)\right)\right|+\left|\varphi_{k}\left(x\left(t_{k}\right)\right)\right|\right)\right] \\
& +t_{k} I_{q_{k}}^{\alpha}|f(t, x(t))| \\
\leq & \frac{\left(t-t_{k}\right)^{\alpha-2}}{\Gamma_{q_{k}}(\alpha-1)}\left[|\beta| t_{k}+\sum_{0<t_{k}<t} \sum_{0<t_{j}<t_{k}}\left(t_{k}-t_{k-1}\right)\right. \\
& \times\left({ }_{t_{j-1}} I_{q_{j-1}}^{1}(|f(s, x(s))-f(s, 0)|+|f(s, 0)|)\left(t_{j}\right)\right. \\
& \left.+\left(\left|\varphi_{j}\left(x\left(t_{j}\right)\right)-\varphi_{j}(0)\right|+\left|\varphi_{j}(0)\right|\right)\right) \\
& +\sum_{0<t_{k}<t}\left\{t_{k-1} I_{q_{k-1}}^{2}(|f(s, x(s))-f(s, 0)|+|f(s, 0)|)\left(t_{k}\right)\right. \\
& \left.\left.+\left(\left|\varphi_{k}^{*}\left(x\left(t_{k}\right)\right)-\varphi_{k}^{*}(0)\right|+\left|\varphi_{k}^{*}(0)\right|\right)\right\}\right] \\
& +\frac{\left(t-t_{k}\right)^{\alpha-1}}{\Gamma_{q_{k}}(\alpha)}\left[|\beta|+\sum_{0<t_{k}<t}\left(t_{k-1} I_{q_{k-1}}^{1}(|f(s, x(s))-f(s, 0)|+|f(s, 0)|)\left(t_{k}\right)\right.\right. \\
& \left.\left.+\left(\left|\varphi_{k}\left(x\left(t_{k}\right)\right)-\varphi_{k}(0)\right|+\left|\varphi_{k}(0)\right|\right)\right)\right]+t_{k} I_{q_{k}}^{\alpha}(|f(s, x(s))-f(s, 0)|+|f(s, 0)|)(t) \\
\leq & \frac{\left(t-t_{k}\right)^{\alpha-2}}{\Gamma_{q_{k}}(\alpha-1)}\left[|\beta| t_{k}+\left(M^{*} R+\Omega_{3}\right) k+\left(L R+\Omega_{1}\right) \sum_{j=1}^{k}\left(t_{j}-t_{j-1}\right) t_{j-1}\right. \\
& \left.+\left(M R+\Omega_{2}\right) \sum_{j=1}^{k}\left(t_{j}-t_{j-1}\right)(j-1)+\left(L R+\Omega_{1}\right) \sum_{j=1}^{k} \frac{\left(t_{j}-t_{j-1}\right)^{2}}{1+q_{j-1}}\right] \\
& +\frac{\left(t-t_{k}\right)^{\alpha-1}}{\Gamma_{q_{k}}(\alpha)}\left[|\beta|+\left(L R+\Omega_{1}\right) t_{k}+\left(M R+\Omega_{2}\right) k\right]+\frac{\left(t-t_{k}\right)^{\alpha}}{\Gamma_{q_{k}}(\alpha+1)}\left(L R+\Omega_{1}\right) .
\end{aligned}
$$

Multiplying both sides of the above inequality by $\left(t-t_{k}\right)^{\gamma}$ for $t \in J_{k}$, we have

$$
\begin{aligned}
(t- & \left.t_{k}\right)^{\gamma}|(\mathcal{Q} x)(t)| \\
\leq & \frac{\left(t-t_{k}\right)^{\gamma+\alpha-2}}{\Gamma_{q_{k}}(\alpha-1)}\left[|\beta| t_{k}+\left(M^{*} R+\Omega_{3}\right) k+\left(L R+\Omega_{1}\right) \sum_{j=1}^{k}\left(t_{j}-t_{j-1}\right) t_{j-1}\right. \\
& \left.+\left(M R+\Omega_{2}\right) \sum_{j=1}^{k}\left(t_{j}-t_{j-1}\right)(j-1)+\left(L R+\Omega_{1}\right) \sum_{j=1}^{k} \frac{\left(t_{j}-t_{j-1}\right)^{2}}{1+q_{j-1}}\right] \\
& +\frac{\left(t-t_{k}\right)^{\gamma+\alpha-1}}{\Gamma_{q_{k}}(\alpha)}\left[|\beta|+\left(L R+\Omega_{1}\right) t_{k}+\left(M R+\Omega_{2}\right) k\right]+\frac{\left(t-t_{k}\right)^{\gamma+\alpha}}{\Gamma_{q_{k}}(\alpha+1)}\left(L R+\Omega_{1}\right) \\
\leq & \Psi_{1} R+\Psi_{2} \leq(\delta+1-\varepsilon) R \leq R,
\end{aligned}
$$

which yields $\|\mathcal{Q} x\|_{P C_{\gamma}} \leq R$. Then we get $\mathcal{Q} B_{R} \subset B_{R}$.

For any $x, y \in P C_{\gamma}(J, \mathbb{R})$ and for each $t \in J_{k}$, we have

$$
\begin{aligned}
& |(\mathcal{Q} x)(t)-(\mathcal{Q} y)(t)| \\
& \quad \leq \frac{\left(t-t_{k}\right)^{\alpha-2}}{\Gamma_{q_{k}}(\alpha-1)}\left[k M^{*}\|x-y\|_{P C_{\gamma}}+L\|x-y\|_{P C_{\gamma}} \sum_{j=1}^{k}\left(t_{j}-t_{j-1}\right) t_{j-1}\right.
\end{aligned}
$$




$$
\begin{aligned}
& \left.+M\|x-y\|_{P C_{\gamma}} \sum_{j=1}^{k}\left(t_{j}-t_{j-1}\right)(j-1)+L\|x-y\|_{P C_{\gamma}} \sum_{j=1}^{k} \frac{\left(t_{j}-t_{j-1}\right)^{2}}{1+q_{j-1}}\right] \\
& +\frac{\left(t-t_{k}\right)^{\alpha-1}}{\Gamma_{q_{k}}(\alpha)}\left[L t_{k}\|x-y\|_{P C_{\gamma}}+k M\|x-y\|_{P C_{\gamma}}\right]+\frac{\left(t-t_{k}\right)^{\alpha}}{\Gamma_{q_{k}}(\alpha+1)} L\|x-y\|_{P C_{\gamma}} .
\end{aligned}
$$

Again multiplying both sides of the above inequality by $\left(t-t_{k}\right)^{\gamma}$ for $t \in J_{k}$, we have

$$
\begin{aligned}
& \left|\left(t-t_{k}\right)^{\gamma}(\mathcal{Q} x)(t)-\left(t-t_{k}\right)^{\gamma}(\mathcal{Q} y)(t)\right| \\
& \leq \frac{\left(t-t_{k}\right)^{\gamma+\alpha-2}}{\Gamma_{q_{k}}(\alpha-1)}\left[k M^{*}\|x-y\|_{P C_{\gamma}}+L\|x-y\|_{P C_{\gamma}} \sum_{j=1}^{k}\left(t_{j}-t_{j-1}\right) t_{j-1}\right. \\
& \left.\quad+M\|x-y\|_{P C_{\gamma}} \sum_{j=1}^{k}\left(t_{j}-t_{j-1}\right)(j-1)+L\|x-y\|_{P C_{\gamma}} \sum_{j=1}^{k} \frac{\left(t_{j}-t_{j-1}\right)^{2}}{1+q_{j-1}}\right] \\
& \quad+\frac{\left(t-t_{k}\right)^{\gamma+\alpha-1}}{\Gamma_{q_{k}}(\alpha)}\left[L t_{k}\|x-y\|_{P C_{\gamma}}+k M\|x-y\|_{P C_{\gamma}}\right]+\frac{\left(t-t_{k}\right)^{\gamma+\alpha}}{\Gamma_{q_{k}}(\alpha+1)} L\|x-y\|_{P C_{\gamma}} \\
& \leq \Psi_{1}\|x-y\|_{P C_{\gamma}},
\end{aligned}
$$

which implies that $\|\mathcal{Q} x-\mathcal{Q} y\|_{P C_{\gamma}} \leq \Psi_{1}\|x-y\|_{P C_{\gamma}}$. As $\Psi_{1}<1$, by the Banach contraction mapping principle, $\mathcal{Q}$ has a fixed point which is a unique solution of (4.7) on $J$.

Example 4.6 Consider the following impulsive fractional $q$-difference initial value problem:

$$
\left\{\begin{array}{l}
t_{k} D_{\left(\frac{k^{3}-3 k+7}{2 k^{4}+k+8}\right)}^{\frac{3}{2}} x(t)=\frac{e^{-\cos ^{2} \pi t}}{3(t+2)^{2}(1+|x(t)|}+\frac{3}{4}, \quad t \in\left[0, \frac{11}{10}\right], t \neq t_{k}, \\
\widetilde{\Delta} x\left(t_{k}\right)=\frac{\left|x\left(t_{k}\right)\right|}{7(k+4)+\left|x\left(t_{k}\right)\right|}, \quad k=1,2, \ldots, 10, t_{k}=\frac{k}{10}, \\
\Delta^{*} x\left(t_{k}\right)=\frac{\left|x\left(t_{k}\right)\right|}{5(k+3)+x\left(t_{k}\right) \mid}, \quad k=1,2, \ldots, 10, t_{k}=\frac{k}{10}, \\
x(0)=0, \quad{ }_{0} D_{\frac{1}{8}}^{\frac{1}{2}} x(0)=\frac{2}{3} .
\end{array}\right.
$$

Here $\alpha=3 / 2, q_{k}=\left(k^{3}-3 k+7\right) /\left(2 k^{4}+k+8\right), k=0,1,2, \ldots, 10, m=10, T=11 / 10, \beta=2 / 3$, $f(t, x)=\left(\left(e^{-\cos ^{2} \pi t}|x|\right) /\left(3(t+2)^{2}(1+|x|)\right)\right)+(3 / 4), \varphi_{k}(x)=(|x|) /(7(k+4)+|x|)$, and $\varphi_{k}^{*}(x)=$ $(|x|) /(5(k+3)+|x|)$. Since $|f(t, x)-f(t, y)| \leq(1 / 12)|x-y|,\left|\varphi_{k}(x)-\varphi_{k}(y)\right| \leq(1 / 35)|x-y|$, and $\left|\varphi_{k}^{*}(x)-\varphi_{k}^{*}(y)\right| \leq(1 / 20)|x-y|$, we have $\left(\mathrm{H}_{1}\right),\left(\mathrm{H}_{2}\right)$, and $\left(\mathrm{H}_{3}\right)$ are satisfied with $L=(1 / 12)$, $M=(1 / 35), M^{*}=(1 / 20)$. Choosing $\gamma=9 / 11$ and using the Maple program, we find that $\widetilde{T}=1.247256483, \widetilde{\Gamma}=0.8934887059$, and

$$
\begin{aligned}
\Psi_{1}= & \widetilde{T} \\
\widetilde{\Gamma} & {\left[m\left(M+M^{*}\right)+L\left(t_{m}+1\right)+L \sum_{j=1}^{m}\left(t_{j}-t_{j-1}\right) t_{j-1}\right.} \\
& \left.+L \sum_{j=1}^{m} \frac{\left(t_{j}-t_{j-1}\right)^{2}}{1+q_{j-1}}+M \sum_{j=1}^{m}\left(t_{j}-t_{j-1}\right)(j-1)\right] \\
\approx & 0.9429923053<1 .
\end{aligned}
$$

Hence, by Theorem 4.5, the initial value problem (4.17) has a unique solution on $[0,11 / 10]$. 


\section{Competing interests}

The authors declare that they have no competing interests.

\section{Authors' contributions}

All authors contributed equally in this article. They read and approved the final manuscript.

\section{Author details}

${ }^{1}$ Nonlinear Dynamic Analysis Research Center, Department of Mathematics, Faculty of Applied Science, King Mongkut's University of Technology North Bangkok, Bangkok, 10800, Thailand. ²Department of Mathematics, University of loannina, loannina, 451 10, Greece. ${ }^{3}$ Department of Mathematics, Anand International College of Engineering, Jaipur, 303012, India.

\section{Authors' information}

Sotiris K Ntouyas is a member of Nonlinear Analysis and Applied Mathematics (NAAM)-Research Group at King Abdulaziz University, Jeddah, Saudi Arabia.

\section{Acknowledgements}

The first author would like to thank Anand International College of Engineering, Jaipur, India, for accommodation while visiting the third author during July 02-10, 2014

\section{Received: 13 August 2014 Accepted: 25 December 2014 Published online: 30 January 2015}

\section{References}

1. Kac, V, Cheung, P: Quantum Calculus. Springer, New York (2002)

2. Jackson, FH: q-Difference equations. Am. J. Math. 32, 305-314 (1910)

3. Al-Salam, WA: Some fractional q-integrals and q-derivatives. Proc. Edinb. Math. Soc. 15(2), 135-140 (1966/1967)

4. Agarwal, RP: Certain fractional $q$-integrals and q-derivatives. Proc. Camb. Philos. Soc. 66, 365-370 (1969)

5. Ernst, T: The history of $q$-calculus and a new method. UUDM Report 2000:16. Department of Mathematics, Uppsala University (2000)

6. Ferreira, RAC: Nontrivial solutions for fractional q-difference boundary value problems. Electron. J. Qual. Theory Differ. Equ. 2010, 70 (2010)

7. Annaby, MH, Mansour, ZS: q-Fractional Calculus and Equations. Lecture Notes in Mathematics, vol. 2056. Springer, Berlin (2012)

8. Bangerezako, G: Variational q-calculus. J. Math. Anal. Appl. 289, 650-665 (2004)

9. Dobrogowska, A, Odzijewicz, A: Second order q-difference equations solvable by factorization method. J. Comput. Appl. Math. 193, 319-346 (2006)

10. Gasper, G, Rahman, M: Some systems of multivariable orthogonal q-Racah polynomials. Ramanujan J. 13, 389-405 (2007)

11. Ismail, MEH, Simeonov, P: q-Difference operators for orthogonal polynomials. J. Comput. Appl. Math. 233, 749-761 (2009)

12. Bohner, M, Guseinov, GS: The $h$-Laplace and q-Laplace transforms. J. Math. Anal. Appl. 365, 75-92 (2010)

13. El-Shahed, M, Hassan, HA: Positive solutions of q-difference equation. Proc. Am. Math. Soc. 138, 1733-1738 (2010)

14. Ahmad, B: Boundary-value problems for nonlinear third-order q-difference equations. Electron. J. Differ. Equ. 2011, 94 (2011)

15. Ahmad, B, Alsaedi, A, Ntouyas, SK: A study of second-order q-difference equations with boundary conditions. Adv. Differ. Equ. 2012, 35 (2012)

16. Ahmad, B, Ntouyas, SK, Purnaras, IK: Existence results for nonlinear q-difference equations with nonlocal boundary conditions. Commun. Appl. Nonlinear Anal. 19, 59-72 (2012)

17. Ahmad, B, Nieto, Jj: On nonlocal boundary value problems of nonlinear q-difference equations. Adv. Differ. Equ. 2012, 81 (2012)

18. Ahmad, B, Ntouyas, SK: Boundary value problems for q-difference inclusions. Abstr. Appl. Anal. 2011, 292860 (2011)

19. Zhou, W, Liu, H: Existence solutions for boundary value problem of nonlinear fractional q-difference equations. Adv. Differ. Equ. 2013, 113 (2013)

20. Yu, C, Wang, J: Existence of solutions for nonlinear second-order $q$-difference equations with first-order $q$-derivatives. Adv. Differ. Equ. 2013, 124 (2013)

21. Tariboon, J, Ntouyas, SK: Quantum calculus on finite intervals and applications to impulsive difference equations. Adv. Differ. Equ. 2013, 282 (2013)

22. Tariboon, J, Ntouyas, SK: Quantum integral inequalities on finite intervals. J. Inequal. Appl. 2014, 121 (2014)

23. Rajković, PM, Marinković, SD, Stanković, MS: Fractional integrals and derivatives in q-calculus. Appl. Anal. Discrete Math. 1, 311-323 (2007)

24. Rajković, PM, Marinković, SD, Stanković, MS: On q-analogues of Caputo derivative and Mittag-Leffler function. Fract. Calc. Appl. Anal. 10(4), 359-373 (2007)

25. Gasper, G, Rahman, M: Basic Hypergeometric Series, 2nd edn. Cambridge University Press, Cambridge (2004) 